\title{
Effects of Shuttlecock-Playing on Physical Fitness in College Students
}

\author{
Tingran Zhang ${ }^{1}$, Jiong Luo ${ }^{1}$ \\ College of Physical Education, Southwest University, 400715, Chongqing, China
}

\author{
Received: October 20, 2014 Accepted: November 10, 2014 Online Published: December 5, 2014 \\ doi:10.11114/jets.v3i1.563 \\ URL:http://dx.doi.org/10.11114/jets.v3i1.563
}

\begin{abstract}
The purpose of this study was to investigate the exercise intensity and the physical fitness effect of shuttlecock playing. 18 normal body weight college students voluntarily participated in this study. They were randomly assigned to shuttlecock playing (SCP) and control groups. The SCP underwent a 15-week shuttlecock-playing program, but the control group did not receive any special exercise activities. The program was arranged as follows: SCP underwent 4 sessions/week, 3 sets/session, $10 \mathrm{~min} / \mathrm{set}$, and rest $5 \mathrm{~min}$ between set. The results indicated that systolic pressure, abdominal skinfold, continuing work time, and hip joint flexibility of SCP were significantly improved after 12-week training. It has been concluded that a 15 -week shuttlecock-playing program could remarkably promote the physical fitness for college students in body characteristics, body flexibility, and aerobic fitness but not muscular strength in lower limbs.
\end{abstract}

Keywords: shuttlecock -playing, physical fitness, college students

\section{Introduction}

A few years ago, Chinese preferred to choose the modern sports introduced from the west. But in recent years, under the tide of fitness for all, a variety of traditional folk sports become more popular, and more and more people choose the traditional forms of physical training. In this context, the scientific research on a variety of traditional folk sports has become hot, and there are more than 70 papers about the effect of traditional folk sports on physical fitness up to now. Feng Zhengqi et al (2013) have studied effects of different schools of Tai Chi on cardiovascular function. Han Renying (2006) has found that Yangko dance has obvious improvement effect on body shape and function of middle aged and old women. Li Jun (2003) has found that playing diabolo can improve antioxidant ability in the elderly. Cui Yongsheng (2004) made the research and the results showed that five-animal exercises could improve cardio-pulmonary function in elderly women. Shuttlecock-playing is widely circulated in China, and edited into physical education of the Elementary and Middle Schools as a kind of traditional folk sports. But there are few domestic studies on it, and mainly focus on the cultural development, such as Tang Yan (2010) pointed out shuttlecock-playing technology, the philosophical foundation of which was the "body of knowledge" philosophy in ancient Chinese, reflecting the concept of harmony between man and nature and the balance of yin and yang in the "body of knowledge" philosophy. This study aims to investigate the effect of shuttlecock-playing on physical fitness and conduct more scientific assessment on exercise value of the traditional folk sports shuttlecock-playing, so as to provide a reference for people choosing a proper form of exercise.

\section{Methods}

\subsection{Subjects}

Sixteen male volunteers from Southwest University participated in this study (age, $21.6 \pm 2.2$ yrs; height, $173.3 \pm 5.2 \mathrm{~cm}$; body weight, $66.2 \pm 5.5 \mathrm{~kg}$ ). All subjects can play shuttlecock 35 times $/ \mathrm{min}$. They were randomly assigned into shuttlecock-playing group and control group, 8 people each group.

\subsection{Methods}

\subsubsection{Experimental Procedure}

The study lasted 15 weeks, and all subjects played shuttlecock at 17:30 on Tuesday, Thursday and Saturday. The movement of playing shuttlecock was defined as kicking shuttlecock at the inside foot. The exercise protocol was as follow: playing shuttlecock $10 \mathrm{~min}$., resting $5 \mathrm{~min}$., playing shuttlecock $10 \mathrm{~min}$., resting $5 \mathrm{~min}$., and playing shuttlecock $10 \mathrm{~min}$. . The total time of shuttlecock-playing was $30 \mathrm{~min}$. 


\subsubsection{Exercise Intensity}

Exercise intensity was controlled by metronome in this study. Shuttlecock-playing was 35 times/min from the first week to the end of the fifth week, 40 times /min from the sixth week to the end of the tenth week, and 45 times/min from the eleventh week to the end of the fifteenth week.

\subsubsection{Experimental Evaluation}

Physical fitness evaluation will be performed on the subjects one week before and after experiment. The testing index was as follows:

Body composition:

1) Body fat percentage, body density were tested using Body Composition Analyzer (Korean Inbody 3.0).

2) Skinfold: chest, waist, and thigh skinfold were tested using skinfold tester.

Physical function:

1) Heart rate: resting heart rate was measured. Japan Casio HEM605 was used to detect 60 seconds resting heart rate of subjects at lying position on the day of playing shuttlecock.

2) Blood pressure: resting blood pressure was measured by Japan Casio HEM605 sphygmomanometer at 15 minutes after sitting quietly.

3) Exercise oxygen consumption: Participants took aerobic fitness test with Sweden cycle ergometer, starting the exercise load of $80 \mathrm{~W}$, increasing $25 \mathrm{~W}$ every 2 minutes. Exhaled air was connected to the cardio-pulmonary breath analysis system through an air pipe for analyzing and recording. During this exercise test, the energy metabolic analytic system was used to monitor, analyze and record breathing oxygen consumption, breathing oxygen consumption per kilogram of body weight and respiratory quotient every 10 seconds. When meeting the following three conditions, breathing oxygen consumption per kilogram of body weight could be determined as exercise maximum oxygen consumption: (1) the exercise load continued to increase but increased oxygen consumption was less than $2 \mathrm{ml} / \mathrm{kg}$. (2) The respiratory quotient>1.1. (3) If participants continue to take exercise, oxygen consumption began to decline.

4) Movement duration and final load: record total duration of exercise on cycle ergometer and the final exercise load when participants feel exhausted and cannot continue pedaling bicycle.

5) Muscle strength of lower extremity: in this study, vertical jump of single leg as bending knee was used to detect single stepping power for assessment of muscle strength of lower extremity. The subjects stood at domestic JP6060 multidimensional force measuring platform, took akimbo posture to maintain body balance, and then squatted with single leg bending for 90 degrees, jumped up with maximum strength, and finally touched the ground with one foot. Video image analysis software was used to calculate the distance of center of gravity from the ground to show muscle strength of lower extremity. The further the distance, the more the strength (Borg, 1962; Davis \& Turner,2011). The dominant side and non-dominant side took 3 effective values, respectively, and the mean was calculated.

6) Lower extremity muscle endurance: the subjects stood at the multidimensional force measuring platform, jumped upward with both legs continuously bended within 30 seconds. Three dimensional stress analysis software was used to record pedaling force at repetitive jumping. The calculation formula of fatigue index: fatigue index $(\%)=($ sum of former 5 measurements - sum of latter 5 measurements / (sum of former 5 measurements $\times 100 \%$ ). Fatigue index of lower extremity can reflect the lower extremity muscle endurance; the lower value indicated better muscle endurance (Gwinup, 1987).

7) Flexibility of hip joint: flexion angles of hip joint can evaluate flexibility of hip joint. The greater joint flexion angles indicated greater articular range of motion that flexibility of hip joint was better Wang et al., 2004). Effective values were taken at dominant side and non-dominant side.

8) Body balance ability test: This study adopted single foot support standing with eyes closed (like a crane standing on its feet) test and lateral step jump balance test to evaluate body balance ability.

Single foot support standing with eyes closed (like a crane standing on its feet) test: the subjects began at biped erect posture, lifted heel and stood on one forefoot, the other leg stretched out and lifted upward, the sole of foot attached to medical side of knee for the support legs, arms posture is not restricted. Standing time of single foot was calculated, and stopped as the forefoot of supporting leg moved forward. The measurement value is accurate to $0.1 \mathrm{~S}$. The mean value was calculated for 3 measurements.

9) Lateral step jump balance test: the subjects stood with single foot at the $X$ point (the body side at A point), jumped to right or left to Point A. The subjects stood at forefoot to maintain balance for 5 seconds, and then inclined forward to push aside the small piece of wood at Point C or Point B within 2 seconds, while heel of supporting foot and the other 
foot could not touch ground. Left and right feet should be measured 2 times for each subject. Five points will be given if the sidefoot exactly stepped on Point A. Five points will be given if the subjects get balance when touching ground and push aside the small piece of wood within 2 seconds. Maintaining balance at Point A for 1 second could get 1 point, and the maximum was 5 points. The full score was 15 points for each time, and 60 points for 4 tests.

2.2.4 Statistical Analysis

SPSS13.0 for Windows was used to make descriptive analysis, paired sample T test, etc. Statistical significant level was set at $\alpha=0.05$.

\section{Results}

\subsection{Repeated Measurements}

For all 16 subjects, all test indexes were measured 2 times except heart rate. All data were detected again, and evaluated with correlation coefficient and variable degree, the correlation coefficient was greater than 0.79 , coefficient of variation was within 5\%, and both reached significant level. Therefore, it can be concluded that the following parameters have higher credibility to analyze body balance ability.

\subsection{Changes of Body Composition before and after Experiment}

As showed in Table 1, the findings were drawn as follows:

1) There was no significant difference in body composition between shuttlecock-playing group and control group before experiment.

2) There was no significant difference in body weight, BMI or body density in shuttlecock-playing group and control group before and after experiment.

3) There was significant difference in body fat percentage, chest, abdominal, and thigh skinfold in shuttlecock-playing group before and after experiment, but no significant difference in body fat percentage, chest, abdominal, and thigh skinfold in control group before and after experiment.

4) There were significant differences in body fat percentage, chest, abdominal, and thigh skinfold between control group and shuttlecock-playing group after experiment.

Table 1. Comparison of body composition before and after experiment

\begin{tabular}{lllllllll}
\hline Variables & \multicolumn{2}{l}{ Shuttlecock-playing } & ( & a $)$ & \multicolumn{2}{l}{ Control } & (b) & \multicolumn{3}{l}{ Test } \\
& Pre & Post & t & Pre & Post & t & Pre & Post \\
\hline Body weight $(\mathrm{kg})$ & $64.2 \pm 4.9$ & $62.7 \pm 4.6$ & 0.64 & $65.35 \pm 6.28$ & $65.4 \pm 6.3$ & 0.58 & Pab & Pab \\
BMI & $21.4 \pm 1.9$ & $20.9 \pm 1.9$ & 0.71 & $21.4 \pm 1.7$ & $21.4 \pm 1.7$ & 0.63 & Pab & Pab \\
body fat (\%) & $16.3 \pm 2.3$ & $14.5 \pm 2.3$ & $2.41^{*}$ & $16.7 \pm 2.9$ & $16.8 \pm 3.3$ & 0.50 & Pab & Pab* \\
Body density & $1.1 \pm 0.1$ & $1.15 \pm 0.15$ & 0.28 & $1.14 \pm 0.18$ & $1.12 \pm 0.21$ & 0.34 & Pab & Pab \\
Chest skinfold (mm) & $11.5 \pm 4.4$ & $9.8 \pm 3.9$ & $2.13^{*}$ & $8.7 \pm 4.5$ & $8.4 \pm 3.2$ & 0.79 & Pab & Pab* \\
Waist skinfold (mm) & $15.2 \pm 6.1$ & $12.8 \pm 5.5$ & $2.65^{*}$ & $10.6 \pm 4.4$ & $11.4 \pm 4.5$ & 0.53 & Pab & Pab* \\
Thigh skinfold (mm) & $12.1 \pm 2.8$ & $10.5 \pm 2.9$ & $2.41^{*}$ & $12.3 \pm 4.3$ & $12.9 \pm 5.1$ & 0.26 & Pab & Pab* \\
\hline
\end{tabular}

* indicated significant difference at $\mathrm{p}<0.05$

\subsection{Changes of Body Function before and After Experiment}

As showed in Table 2, the findings were drawn as follows:

1) There was no significant difference in each index between shuttlecock-playing group and control group before experiment.

2) There was no significant difference in each index for control group before and after experiment.

3) There was no significant difference in muscle strength of lower extremity for shuttlecock-playing group before and after experiment.

4) There were significant differences in systolic pressure, pedaling time, flexion angles of bilateral hip joints, single foot support standing test, lateral step jump balance test for shuttlecock-playing group before and after experiment, and differences were also found in these indexes between shuttlecock-playing group and control group. 
Table 2. Comparison of body function before and after experiment

\begin{tabular}{|c|c|c|c|c|c|c|c|c|}
\hline & \multicolumn{2}{|c|}{ Shuttlecock-playing （a） } & \multicolumn{3}{|c|}{ Control （b） } & \multicolumn{3}{|c|}{ Test } \\
\hline & Pre & Post & $\mathrm{t}$ & Pre & Post & $\mathrm{t}$ & Pre & Post \\
\hline \multicolumn{9}{|l|}{ Aerobic ability } \\
\hline Resting heart rate (bpm) & $69.8 \pm 7.5$ & $69.0 \pm 8.8$ & 1.13 & $74.1 \pm 7.3$ & $71.3 \pm 8.1$ & -0.87 & $\mathrm{Pab}$ & $\mathrm{Pab}$ \\
\hline Systolic pressure $(\mathrm{mmHg})$ & $123.0 \pm 13.2$ & $110.3 \pm 13.4$ & $3.94 *$ & $113.2 \pm 12.3$ & $108.1 \pm 5.6$ & -1.85 & $\mathrm{Pab}$ & $\mathrm{Pab}^{*}$ \\
\hline Diastolic pressure $(\mathrm{mmHg})$ & $68.0 \pm 12.6$ & $64.5 \pm 8.7$ & 1.52 & $66.0 \pm 8.3$ & $65.5 \pm 6.1$ & -0.23 & $\mathrm{Pab}$ & $\mathrm{Pab}$ \\
\hline $\mathrm{VO} 2 \mathrm{max}(\mathrm{ml} / \mathrm{kg} / \mathrm{min})$ & $39.3 \pm 6.9$ & $42.5 \pm 10.1$ & 0.97 & $38.7 \pm 8.9$ & $35.7 \pm 7.2$ & -0.76 & $\mathrm{Pab}$ & $\mathrm{Pab}$ \\
\hline Pedaling load（watt） & $186.3 \pm 32.0$ & $192.5 \pm 23.1$ & 0.78 & $180.6 \pm 29.9$ & $176.9 \pm 33.9$ & -0.87 & $\mathrm{Pab}$ & $\mathrm{Pab}$ \\
\hline Pedaling time $(\mathrm{sec})$ & $565.8 \pm 146.0$ & $616.3 \pm 122.2$ & $2.76^{*}$ & $530.1 \pm 142.6$ & $510.0 \pm 168.4$ & -1.22 & $\mathrm{Pab}$ & $\mathrm{Pab}^{*}$ \\
\hline \multicolumn{9}{|l|}{ Muscle strength of lower extremity } \\
\hline Jumping distance $($ dominant $)(\mathrm{cm})$ & $29.6 \pm 5.6$ & $30.2 \pm 6.7$ & 1.34 & $28.4 \pm 7.9$ & $27.2 \pm 5.3$ & 0.73 & $\mathrm{Pab}$ & $\mathrm{Pab}$ \\
\hline Jumping distance $($ non-dominant $)(\mathrm{cm})$ & $29.7 \pm 5.7$ & $35.3 \pm 6.4$ & 0.93 & $29.6 \pm 9.2$ & $27.6 \pm 6.2$ & -1.24 & $\mathrm{Pab}$ & $\mathrm{Pab}$ \\
\hline Fatigue index $(\%)$ & $0.8 \pm 4.9$ & $87.7 \pm 10.7$ & 1.24 & $89.1 \pm 8.8$ & $88.5 \pm 6.3$ & -1.22 & $\mathrm{Pab}$ & $\mathrm{Pab}$ \\
\hline \multicolumn{9}{|l|}{ Body flexibility } \\
\hline Flexion angle of hip joint (dominant) & $103.9 \pm 8.2$ & $118.6 \pm 13.5$ & $2.87 *$ & $108.7 \pm 16.6$ & $109.6 \pm 8.6$ & 0.85 & $\mathrm{Pab}$ & $\mathrm{Pab}^{*}$ \\
\hline $\begin{array}{l}\text { Flexion angel of hip joint } \\
\text { (non-dominant) }\end{array}$ & $107.9 \pm 9.3$ & $117.9 \pm 13.5$ & $3.41 *$ & $103.4 \pm 12.4$ & $105.4 \pm 7.4$ & 0.92 & $\mathrm{Pab}$ & $\mathrm{Pab}^{*}$ \\
\hline \multicolumn{9}{|l|}{ Balance ability } \\
\hline Single foot support standing $(\mathrm{s})$ & $18.3 \pm 5.4$ & $45.1 \pm 4.0$ & $4.57 *$ & $16.9 \pm 3.2$ & $18.3 \pm 2.7$ & 0.98 & $\mathrm{Pab}$ & $\mathrm{Pab}^{*}$ \\
\hline Lateral step jump balance & $33 \pm 9$ & $53 \pm 7$ & $3.90 *$ & $36 \pm 5$ & $40 \pm 7$ & 0.94 & $\mathrm{Pab}$ & $\mathrm{Pab}^{*}$ \\
\hline
\end{tabular}

* indicated significant difference at $\mathrm{p}<0.05$

\section{Discussion}

\subsection{Analysis of Body Composition Changes}

As indicated in table 1, there was no remarkably difference in each index between shuttlecock-playing group and control group before experiment, which indicated that body composition were consistent for subjects in both two groups at the beginning of experiment. After 15 weeks of experiment, no significant difference was found in body weight, BMI or body density for subjects in shuttlecock-playing group or control group before and after experiment. The results indicated that shuttlecock-playing did not significantly change body weight of subjects in shuttlecock-playing group, which consisted to the result of taking aerobic exercise with 60-80\% exercise intensity for 8-12 weeks to influencing body weight by Kieres (1991). The reasons for unobvious change of body weight may be that BMI was within normal range for subjects in control group and shuttlecock-playing group before training, and all subjects were not on a diet during the whole experiment. Hoeger \& Hoeger (2002) put forward the opinion that it was more difficult to reduce body weight and body fat percentage for people with normal BMI than overweight people. Winup(1987) thought that diet control was the essential factor in exercise weight loss intervention experiment. There were significant differences in body fat percentage, chest, waist and thigh skinfold for subjects in shuttlecock-playing group before and after experiment. Siri (1961) found that body weight was the sum of fat weight and removing fat weight. If body weight was constant and fat weight reduces, the removing fat weight increases. Generally speaking, change of removing fat weight can represent the change of muscle. Shuttlecock-playing was a kind of moderate to low intensity exercise. This paper's result of body fat percentage was consistent with that of 12 weeks of aerobic exercise on body composition by Zhang Meili, so the mechanism of reducing body fat for shuttlecock-playing was similar to that of aerobic exercise. Powers \& Howley (2001) indicated that it was slow for sports changing body composition and the extent was modest. Davis \& Turner (2011) proposed more than 3 times of aerobic exercise for over 20 minutes in a week and continuous for 3 months can obviously reduce body fat percentage in 92\% subjects. Unger (2003) believed that people with accumulation of fat in the waist had higher risk of having high blood lipids, hypertension, diabetes, high uric acid and other metabolic syndrome than those with accumulation of fat in buttock and legs, so shuttlecock-playing can improve body composition and promote body health even without diet control for subjects in shuttlecock-playing group.

\subsection{Analysis of Body Function Changes}

As indicated in table 2, no significant difference was found in aerobic capacity, muscle strength of lower extremity, 
flexibility or body balance ability between shuttlecock-playing group and control group before experiment. No significant difference was found in aerobic capacity, muscle strength of lower extremity, flexibility or body balance ability for subjects in control group before and after experiment. There were significant differences in systolic pressure, pedaling time, flexion angles of bilateral hip joints, single foot support standing test, lateral step jump balance test for subjects in shuttlecock-playing group before and after experiment, and other evaluation indexes also improved. Resting heart rate, systolic pressure and maximum oxygen consumption and other cardio-pulmonary function indexes were within normal range for all subjects before experiment, but systolic pressure also reduced obviously for subjects in shuttlecock-playing group after experiment, while there was no obvious change of each cardio-pulmonary function index for subjects in control group. Saltin (1969) proposed that reasons for increase of maximal oxygen consumption in young male non-athletes were increases of stroke volume and arteriovenous oxygen difference. The main cause of increased stroke volume is that peripheral vascular resistance decrease after sports, which can reduce blood pressure and increase peripheral blood flow. The research carried out by Klausen et al. (1982) showed that doing moderate intensity exercise by pedaling cycle ergometer with single foot could reduce peripheral vascular resistance, thus had better effect on increasing stroke volume than that of the sports model by two legs. Single leg movement model is similar with shuttlecock-playing by single foot in this study. Therefore, mechanisms of systolic pressure decrease and maximum oxygen consumption increase may be that shuttlecock-playing activities decreases peripheral vascular resistance. Another mechanism may be continuous shuttlecock-playing can improve arteriovenous oxygen difference of lower extremities, and then increase maximal oxygen consumption. In indexes for muscle strength of lower extremities, jumping distance and fatigue index had no obvious change between two groups. For body flexibility, flexion angle of hip joint obviously improved in shuttlecock-playing group, Craik indicated that cross legged motion in yoga had a good effect on improving flexibility of hip joint, and shuttlecock-playing movement was similar with cross legged motion in yoga. Significant difference was found in body balance ability for subjects in shuttlecock-playing group before and after experiment, and shuttlecock-playing movement could obviously improve body balance ability. Wang et al. (2004) believed that single foot support can improve body balance and muscle control of waist and lower extremity. Stefanyshyn et al. (2000) presented a single foot standing for a long time could exercise deep muscle of waist. Ebbeling et al. (1994) found leg-raising to the inside of thigh could exercise abdominal muscle and iliopsoas muscle.

\section{Conclusions}

Shuttlecock-playing can enhance cardio-pulmonary function, decrease body fat content, lower systolic blood pressure and increase body flexibility and balance ability for young adult.

Shuttlecock-playing has little influence on muscle strength of lower extremity in young adult.

Shuttlecock-playing can improve physical fitness of young man. Due to the limitation of experimental conditions, whether shuttlecock-playing training can improve agility and coordination or not remains to be discussed. More studies are also needed to investigate shuttlecock-playing training on exercise intensity and physical fitness for middle-aged people, children, young women or other population.

\section{References}

Borg, G. V. (1962). Physical performance and perceived exertion. Lund, Sweden: Gleerup.

Cernekova, M., \& Hlavacek, P. (2008). The influence of heel height on plantar pressure. Clinical Biomechanics, 23, 667-668. http://dx.doi.org/10.1016/j.clinbiomech.2008.03.008

Cui, Y. S., \& Yu, D. H. (2004). The effect on middle-aged and old women's body and mind by health Qigong Wuqinxi exercise. Journal of Beijing Sport University, 27, 1504-1507.

Davis, R. B., \& Turner, L. W. (2011). A review of current weight management: Research and recommendations. Journal of the American Academy of Nurse Practitioners, 13, 15-19. http://dx.doi.org/10.1111/j.1745-7599.2001.tb00210.x

Ebbeling, C. J., Hamill, J., \& Crussemeyer, J. A. (1994). Lower extremity mechanics and energy cost of walking in high heeled shoes. Journal of Orthopedics Sports Physical Therapy, 19, 190-196. http://dx.doi.org/10.2519/jospt.1994.19.4.190

Feng, Z. Q. (2013). Effects of Chen style and Yang style Taijiquan exercise on cardiovascular function of university students. Journal of Zhengzhou University (Medical Sciences), 48, 253-257.

Gwinup, G. (1987). Weight loss without dietary restriction: Efficacy of different forms of aerobic exercise. American Journal of Sports Medicine, 15, 275-279. http://dx.doi.org/10.1177/036354658701500317

Han, R. Y. (2006). The Effects of Yang-ge Dance on the Figure, Functioning and Physical Qualities of the Middle and Old Aged Female (Unpublished master's theses). Beijing Sport University, Beijing, China.

Hoeger, W., W. K., \& Hoeger, S. A. (2002). Principles and labs for fitness and wellness. Belmont, CA: Wadsworth / 
Thomson Learning, Inc. 122-123.

Kieres, J., \& Plowman, S. (1991). Effects of swimming and land exercises versus swimming and water exercises on composition of college students. The Journal of Sports Medicine and Physical Fitness, 31, 189-195.

Klausen, K., Secher, N. H., Clausen, J. P., Hartling, O., \& Trap-Jensen, J. (1982). Central and regional circulatory adaptations to one-leg training. Journal of Applied Physiology: Respiratory, Environmental and Exercise Physiology, 52, 976-983.

Li, J. (2003). Effect of Diabolo Exercise on anti-aged ability of elder people. China Sport Science and Technology, 42(20): 96-99.

Li, J. X., Hong, Y., \& Chan, K. M. (2001). Tai chi: physiological characteristics and beneficial effects on health. British Journal of Sports Medicine, 35, 148-156. http://dx.doi.org/10.1136/bjsm.35.3.148

Powers, S. K., \& Howley, E. T. (2001). Exercise physiology (4th ed.). New York, N.Y.: McGraw-Hill Companies.

Saltin, B.. (1969). Physiological effects of physical conditioning. Medicine and Science in Sports, 1, 50-56. http://dx.doi.org/10.1249/00005768-196903000-00011

Siri, W. E. (1961). Body composition from fluid spaces and density: Analysis of methods. In J. Brozek \& A. Henschel (Eds.) Techniques for measuring body composition. Washington, D.C. National Academy of Sciences-National Research Council. 22, 223-244.

Stefanyshyn, D. J., Nigg, B. M., Fisher, V. B., \& Liu, W. (2000). The influence of high heeled shoes on kinematics, kinetics, and muscle EMG of normal female gait. Journal of Applied Biomechanic, 16, 309-319.

Tang, Y. (2010). Change of shuttlecock Culture. Sports Culture Guide, 21(7), 108-111.

Unger, R. H. (2003). Minireview: Weapons of lean body mass destruction: The role of ectopic lipids in the metabolic syndrome. Endocrinology, 144, 5159-5165. http://dx.doi.org/10.1210/en.2003-0870

Wang, C., Collet, J. P., \& Lau, J. (2004). The effect of Tai Chi on health outcomes in patients with chronic conditions: A systematic review. Archives of Internal Medicine, 164, 493. http://dx.doi.org/10.1001/archinte.164.5.493

Williams \& Wilkins. American College of Sports Medicine. (2000). ACSM's guidelines for exercise testing and prescription. Baltimore: Lippincott,

\section{(cc) Br}

This work is licensed under a Creative Commons Attribution 3.0 License. 